\title{
Kinetics of growth and sugar consumption in yeasts
}

\author{
Johannes P. van Dijken, Ruud A. Weusthuis \& Jack T. Pronk \\ Department of Microbiology and Enzymology, Kluyver Laboratory of Biotechnology, Julianalaan 67, \\ 2628 BC Delft, The Netherlands
}

Key words: alcoholic fermentation, chemostat culture, Crabtree effect, respiration, Saccharomyces cerevisiae, yeasts

\begin{abstract}
An overview is presented of the steady- and transient state kinetics of growth and formation of metabolic byproducts in yeasts. Saccharomyces cerevisiae is strongly inclined to perform alcoholic fermentation. Even under fully aerobic conditions, ethanol is produced by this yeast when sugars are present in excess. This so-called 'Crabtree effect' probably results from a multiplicity of factors, including the mode of sugar transport and the regulation of enzyme activities involved in respiration and alcoholic fermentation. The Crabtree effect in $S$. cerevisiae is not caused by an intrinsic inability to adjust its respiratory activity to high glycolytic fluxes. Under certain cultivation conditions, for example during growth in the presence of weak organic acids, very high respiration rates can be achieved by this yeast. $S$. cerevisiae is an exceptional yeast since, in contrast to most other species that are able to perform alcoholic fermentation, it can grow under strictly anaerobic conditions.

'Non-Saccharomyces' yeasts require a growth-limiting supply of oxygen (i.e. oxygen-limited growth conditions) to trigger alcoholic fermentation. However, complete absence of oxygen results in cessation of growth and therefore, ultimately, of alcoholic fermentation. Since it is very difficult to reproducibly achieve the right oxygen dosage in large-scale fermentations, non-Saccharomyces yeasts are therefore not suitable for largescale alcoholic fermentation of sugar-containing waste streams. In these yeasts, alcoholic fermentation is also dependent on the type of sugar. For example, the facultatively fermentative yeast Candida utilis does not ferment maltose, not even under oxygen-limited growth conditions, although this disaccharide supports rapid oxidative growth.
\end{abstract}

\section{Introduction}

The yeast collection of the Centraal Bureau voor Schimmelcultures (CBS, Delft, The Netherlands) harbours all (over 4,500) natural yeast isolates described in the literature. These belong to 640 species that have been grouped into 75 genera. Due to its 'classical' industrial applications in the rising of dough and in beer and wine fermentation, Saccharomyces cerevisiae is the best-known representative of this group of microbes. In fact, 'yeast' and S. cerevisiae are frequently used as synonymous terms.
However, $S$. cerevisiae is a rather exceptional yeast since it is one of the few yeasts that are able to grow anaerobically (Visser et al.1990). Also during aerobic growth, this yeast shows an unusual behaviour. When grown aerobically at a low rate, under sugar limitation, cultures tend to spontaneously synchronize their cell cycle (von Meyenburg 1969; Parulekar et al. 1986), which makes the analysis of chemical kinetics of growth a difficult enterprise.

In the past decade, yeasts other than $S$. cerevisiae have gained industrial interest as hosts for the expression of heterologous genes. Examples are 
methanol-utilizing yeasts such as Hansenula polymorph a and Pichia pastoris and the lactose-utilizing species Kluyveromyces lactis and $K$. marxianus (Romanos et al. 1992). Several arguments have been put forward to use 'non-Saccharomyces yeasts' as hosts for heterologous gene expression, including broader substrate specificity, availability of strong inducible promoters, absence of aerobic alcoholic fermentation (i.e. absence of the Crabtree effect), etc. However, a major factor, decisive for the use of alternative yeasts in commercial processes, is correct processing of the heterologous gene product, for example with respect to glycosylation and excretion. In various cases $S$. cerevisiae strains exhibit unwanted traits in this respect. It is presently expected that application of non-Saccharomyces yeasts in large-scale fermentations for the production of heterologous proteins will be common industrial practice before the end of this century. However, it remains to be seen whether all of the presumed advantages of non-Saccharomyces yeasts will still apply in large-scale industrial fermentations.

Large-scale industrial fermentations are characterized by high cell densities (over $50 \mathrm{~g}$ dry weight $\left.\mathrm{I}^{-1}\right)$. Aerobic, substrate-limited cultivation conditions (fed-batch cultivation) are employed in order to achieve optimal biomass yields (both on the carbon substrate and on oxygen), and minimal byproduct formation. However, in large reactors, due to imperfect mixing, gradients in substrate and oxygen cannot be avoided. A better understanding of transient state responses of yeasts is therefore a necessity for their application in large-scale processes.

In this paper, an overview will be given of those aspects of the kinetics of growth and product formation by yeasts that are relevant for their large-scale cultivation. In view of the increasing importance of non-Saccharomyces yeasts as catalysts for production of 'bioalcohol' and as hosts for heterologous gene expression, these organisms will be compared with the 'classical' S. cerevisiae option.

\section{Occurrence of alcoholic fermentation}

Quantitatively the most important byproduct of yeast biomass is, generally speaking, ethanol. It is therefore relevant to classify yeasts with respect to their fermentative abilities (Table 1). Certain yeasts (such as Rhodotorula species) cannot perform alcoholic fermentation, possibly due to their inability to synthesize key enzymes of the fermentative pathway, whereas others (for example Candida slooffi) have to rely on alcoholic fermentation as a source of metabolic energy, since they are by nature unable to respire. The latter phenotype is also observed in a class of frequently occurring $S$. cerevisiae mutants with impaired mitochondrial function, the so-called 'petite' or respiratory-deficient mutants. However, the large majority of the known yeast species are facultatively fermentative, and can thrive on either oxidative or substrate-level phosphorylation as a source of ATP for biosynthesis. The group of facultatively fermentative yeasts is larger than is apparent from handbooks on yeast taxonomy: even the yeast Hansenula nonfermentans can exhibit appreciable rates of alcoholic fermentation under appropriate cultivation conditions (Van Dijken et al. 1986).

It is important to realize that the term 'facultatively fermentative' is not synonymous with 'facultatively anaerobic'. As mentioned in the introduction, only very few yeasts (in particular $S$. cerevisiae) are able to grow in the absence of oxygen. A more detailed discussion of the effects of oxygen on growth and ethanol production by yeasts will be given below.

In addition to oxygen concentration, the concentration of fermentable sugars in yeast cultures can also be a key factor in the regulation of alcoholic

Table 1. Physiological classification of yeasts on the basis of the occurrence of alcoholic fermentation of sugars.

\begin{tabular}{ll}
\hline Classification & Examples \\
\hline Non-fermentative & Rhodotorula glutinis \\
Facultatively fermentative & $\begin{array}{l}\text { a. Crabtree-positive: } \\
\text { Saccharomyces cerevisiae } \\
\end{array}$ \\
& Schizosaccharomyces pombe \\
& b. Crabtree-negative: \\
& Candida utilis \\
& Kluyveromyces marxianus \\
& Candida Slooffi \\
Obligately fermentative & \\
\hline
\end{tabular}




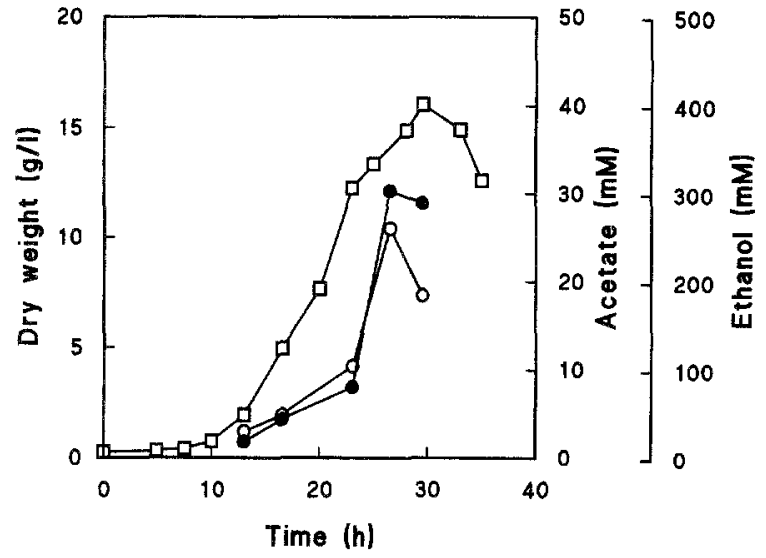

Fig. 1. Deregulation of sugar metabolism in an unsuccesful sucrose-limited fed-batch culture of Kluyveromyces marxianus CBS 6556, resulting in byproduct formation. The observed production of alcohol and acetic acid was not due to oxygen limitation: the DOT was maintained at $20 \%$ of air saturation throughout the fermentation. The decrease of the biomass concentration at the end of the fermentation resulted from dilution of the poorly growing culture by the continous medium feed. Symbols: $\square$, culture dry weight; $\bullet$, ethanol concentration; $O$, acetic acid concentration.

fermentation. Facultatively fermentative yeasts can be subdivided on the basis of the Crabtree effect: the occurrence of alcoholic fermentation under strictly aerobic conditions in the presence of excess sugar (Table 1; Van Dijken \& Scheffers 1986). When sugar-limited cultures of Crabtree-positive yeasts are suddenly exposed to sugar excess (as may for example occur due to imperfect mixing in large reactors) ethanol is produced instantaneously (Verduyn et al. 1984). Production of ethanol is accompanied by the appearance of other metabolites such as acetic acid, pyruvic acid and ethylacetate. The detrimental effects of ethanol on yeast growth include an increased membrane permeability and inhibition of a number of essential enzymes (for a review see D'Amore and Stewart 1987). The effect of weak acids on yeast growth will be discussed below.

The separation of facultatively fermentative yeasts into Crabtree-positive and Crabtree-negative yeasts is not a strict one. Also in yeasts which are regarded as Crabtree-negative, deregulation of metabolism, resulting in the formation of ethanol and other metabolites, can occur under strictly aerobic conditions. For example, in dense cultures (10$130 \mathrm{~g}$ dry weight $\mathrm{1}^{-1}$ ) of Kluyveromyces marxianus, aerobic production of ethanol and organic acids can occur under inappropriate cultivation conditions (Fig. 1). Especially in high cell density cultures, byproduct formation may have detrimental effects since, despite low specific production rates, they may rapidly accumulate to toxic levels. Therefore, the apparent absence of interfering byproducts in small-scale laboratory fermentations (i.e. a 'clean' Crabtree-negative phenotype) does not preclude their accumulation in large-scale, high cell density situations.

\section{Metabolic pathways and byproduct formation}

Whether or not byproduct formation will occur under a given set of environmental conditions depends on the size of various metabolic fluxes which, in turn, are governed by substrate pools and enzyme activities (Fig. 2). Crabtree-positive and Crabtree-negative yeasts exhibit striking differenc-



Fig. 2. Schematic representation of glucose metabolism in yeasts. (1) Glucose transport: facilitated diffusion or active transport; (2) Formation of glycogen and trehalose; (3) Glycolysis; (4) Pyruvate decarboxylase; (5) Alcohol dehydrogenase(s); (6) Acetaldehyde dehydrogenase(s); (7) Acetyl-CoA synthetase (cytoplasmic); (8) Transport of acetyl-CoA into mitochondria via the carnitine shuttle; (9) Transport of acetate into mitochondria and formation of acetyl-CoA via mitochondrial acetyl-CoA synthetase; (10) mitochondrial pyruvate transport; (11) pyruvate dehydrogenase complex; (12) TCA cycle; (13) Assimilation of pyruvate into cell material, initiated by pyruvate carboxylase; (14) ADP/ATP translocator; (15) Formation of ATP via oxidative phosphorylation. 




Fig. 3. Competition between Saccharomyces cerevisiae CBS 8066 $(O)$ and Candida utilis CBS $621(\bullet)$ in a glucose-limited, aerobic chemostat culture grown at $\mathrm{D}=0.10 \mathrm{~h}^{-1}$. At zero time, a steadystate $S$. cerevisiae culture was inoculated with $1 \%$ (dry weight) $C$. utilis cells. The number of viable cells of each yeast is expressed as percentage of the total cell population. Also plotted is the residual glucose concentration in the mixed culture $(\triangle)$.

es with respect to sugar transport and the regulation of fluxes at T-junctions in the metabolism of sugars at the level of sugar phosphates and pyruvate. These are discussed below.

\section{Glucose transport}

When grown under sugar limitation in aerobic chemostat cultures, Crabtree-negative yeasts contain glucose carriers with a high substrate affinity. Sugar transport in these yeasts is an energy-requiring process since such cells accumulate the non-metabolizable analogue 6-deoxyglucose (Table 2; Van Urk et al. 1989a). In Crabtree-positive yeasts on the other hand, transport of glucose occurs via facilitated diffusion and 6-deoxyglucose is not accumulated. The glucose carriers in the latter yeasts have a low affinity for their substrate. This difference in glucose transport between Crabtree-positive and Crabtreenegative yeasts is also reflected in the residual glucose concentrations in sugar-limited chemostat cultures (Table 2), which is higher in cultures of Crabtree-positive yeasts. Consequently, when a sugarlimited culture of a Crabtree-positive yeast becomes contaminated with a Crabtree-negative yeast, the former is rapidly outcompeted (Fig. 3; Postma et al. 1989a). The higher affinity of Crabtree-negative yeasts for glucose explains the problems with so-called 'wild yeasts' in industrial sugarlimited fed-batch cultures of baker's yeast. These contaminants (frequently Candida species) tend to outgrow the commercial $S$. cerevisiae strains and negatively affect product quality.

\section{The pyruvate junction}

At the level of pyruvate, 3 metabolic fluxes can be discriminated (Fig. 2):

1. carboxylation of pyruvate to oxaloacetate to re-

Table 2. Accumulation of [ ${ }^{3} \mathrm{H}-6$-deoxyglucose], apparent affinity constant $\left(\mathrm{K}_{\mathrm{m}}\right)$ for glucose and capacities $\left(\mathrm{V}_{\text {max }}\right)$ of glucose uptake by yeast suspensions, pregrown under aerobic glucose-limited conditions at $\mathrm{D}=0.10 \mathrm{~h}^{-1}$ and the residual glucose concentration in these cultures. Data from Van Urk et al, (1989a).

\begin{tabular}{|c|c|c|c|c|}
\hline & $\begin{array}{l}\text { Accumulation } \\
\text { 6-deoxyglucose }\end{array}$ & $\begin{array}{l}\mathrm{K}_{\mathrm{m}}{ }^{1} \\
\mathrm{mM}\end{array}$ & $\begin{array}{l}\mathrm{V}_{\max }^{1} \\
\mathrm{mmol} \mathrm{g}^{-1} \mathrm{~h}^{-1}\end{array}$ & $\begin{array}{l}\text { Residual glucose } \mathrm{e}^{2} \\
\mu \mathrm{M}\end{array}$ \\
\hline \multicolumn{5}{|l|}{ Crabtree-positive yeasts } \\
\hline Saccharomyces cerevisiae & $0.5 \times$ & $1.0,20$ & 12,9 & 110 \\
\hline Schizosaccharomyces pombe & $1.1 \times$ & 1.5 & 9 & 160 \\
\hline \multicolumn{5}{|l|}{ Crabtree-negative yeasts } \\
\hline Candida utilis & $70 \times$ & $0.025,0.2$ & $8.4,5.4$ & 5 \\
\hline Kluyveromyces marxianus & $110 \times$ & $0.025,1.8$ & $1.2,3.0$ & 35 \\
\hline
\end{tabular}

${ }^{1}$ Determined in 10 sec incubations with $\mathrm{D}-\left[\mathrm{U}_{-}{ }^{14} \mathrm{C}\right]$ glucose. In many cases biphasic Hanes plots were obtained. This is indicated by the two values for $\mathrm{K}_{\mathrm{m}}$ and $\mathrm{V}_{\max }$.

${ }^{2}$ Determined after rapid sampling of cultures into liquid nitrogen. 
plenish the TCA cycle intermediates that are used for biosynthesis;

2. transport of pyruvate into the mitochondrion and subsequent oxidation to acetyl-CoA, which enters the TCA cycle; and

3. decarboxylation of pyruvate by cytoplasmic pyruvate decarboxylase to acetaldehyde.

The $\mathbf{K}_{\mathrm{m}}$ for oxidation of pyruvate by isolated mitochondria is approximately tenfold lower than the $\mathrm{K}_{\mathrm{m}}$ of pyruvate decarboxylase (Van Urk et al. 1989b). Therefore, when the intracellular pyruvate concentration is low, pyruvate is preferentially channeled into the TCA cycle. This particularly holds for Crabtree-negative yeasts, since in these organisms the pyruvate decarboxylase levels are low under aerobic growth conditions. In these yeasts high pyruvate decarboxylase levels are only present during growth under oxygen limitation.

At the level of acetaldehyde, another T-junction occurs: acetaldehyde may be either reduced to ethanol or oxidized to acetate (Fig. 2). The $\mathrm{K}_{\mathrm{m}}$ of the acetaldehyde dehydrogenase for acetaldehyde is 100-fold lower than that of alcohol dehydrogenase (Postma et al. 1989b). Moreover, since the activity of the pyruvate bypass (the metabolic sequence from pyruvate to acetyl-CoA involving the enzymes pyruvate decarboxylase, acetaldehyde dehydrogenase and acetyl-CoA synthetase; Fig. 2) is higher in Crabtree-negative yeasts than in Crabtree-positive yeasts (Van Urk et al. 1990), it is evident that formation of acetyl-CoA rather than etha- nol is the preferred route of glucose metabolism in aerobic cultures of Crabtree-negative yeasts.

\section{Transient state kinetics of sugar metabolism}

Crabtree-positive yeasts immediately produce ethanol and acetate upon transfer from glucose limitation to glucose excess, even under fully aerobic conditions. This so-called 'short-term Crabtree-effect' has been ascribed to the inability of such yeasts to instantaneously enhance their respiration rate (Petrik et al. 1983; Rieger et al. 1983; Käppeli 1986). This would leave them unable to completely respire the increased flux of sugar entering the cell (and the glycolytic pathway) as a consequence of the increased extracellular sugar concentration. However, so far the metabolic 'bottlenecks' responsible for such a limited respiratory capacity have not been identified.

In the equilibrium (steady state) situation, the residual sugar concentration in the culture is at or below the $\mathrm{K}_{\mathrm{m}}$ of the glucose carriers (Table 2). The transition to sugar excess therefore immediately results in an enhanced rate of uptake. However, the rate of sugar consumption during the first $30 \mathrm{~min}$ utes after a glucose pulse is lower than calculated on the basis of the kinetic parameters of the glucose carriers (Table 3). Apparently, after the switch to glucose excess, the rate of sugar transport is adapted, such that it does not operate at full speed. Despite this adaptation, intracellular pyruvate accu-

Table 3. Comparison of the calculated and observed glucose consumption rates during a glucose pulse ( $50 \mathrm{mM})$ to cultures of yeasts pregrown under glucose limitation at a dilution rate of $0.10 \mathrm{~h}^{-1}$. The calculated rate was obtained from the apparent affinity constants and capacities for the glucose carriers as determined in $10 \mathrm{sec}$ incubations with $\mathrm{D}-\left[\mathrm{U}-{ }^{14} \mathrm{C}\right]$ glucose. Also presented are the observed rates of oxygen consumption, ethanol and acetate production during the first $30 \mathrm{~min}$ after the pulse. Rates are expressed as mmol g cells $\mathrm{s}^{-1} \mathrm{~h}^{-1}$. Data from Van Urk et al. (1990).

\begin{tabular}{|c|c|c|c|c|c|}
\hline & \multicolumn{2}{|l|}{ q glucose } & \multirow[t]{2}{*}{$\mathrm{qO}_{2}$} & \multirow[t]{2}{*}{ q ethanol } & \multirow[t]{2}{*}{$\mathrm{q}$ acetate } \\
\hline & Calculated & Observed & & & \\
\hline \multicolumn{6}{|l|}{ Crabtree-positive yeasts } \\
\hline Saccharomyces cerevisiae & 18.0 & 5.4 & 6.0 & 5.6 & 1.3 \\
\hline Schizosaccharomyces pombe & 8.7 & 7.1 & 4.2 & 5.3 & 0.3 \\
\hline \multicolumn{6}{|l|}{ Crabtree-negative yeasts } \\
\hline Candida utilis & 13.8 & 5.0 & 6.6 & 0.05 & 0.05 \\
\hline Kluyveromyces marxianus & 4.2 & 3.5 & 7.1 & 0 & 0 \\
\hline
\end{tabular}




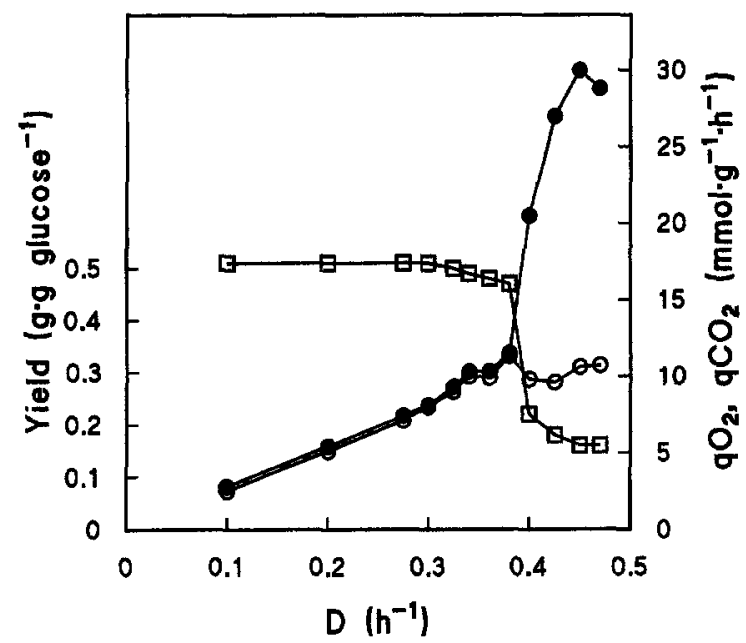

Fig. 4. Specific rates of oxygen uptake $(O)$, carbon dioxide production $(\bullet)$ and cell yield $(\square)$ gram dry weight $\left(g_{\text {glucose }}^{-1}\right.$ ) as a function of the dilution rate in aerobic, glucose-limited cultures of Saccharomyces cerevisiae CBS 8066.

mulation occurs, resulting in an enhanced flux via pyruvate decarboxylase, thereby causing the simultaneous formation of ethanol and acetate in those yeasts that contain high levels of pyruvate decarboxylase and low levels of acetyl-CoA synthetase (Van Urk et al. 1990).

From a comparative study on the behaviour of yeasts after the transition from glucose limitation to glucose excess (Van Urk et al. 1990), it appears that a limited respiratory capacity may not be the primary cause of byproduct formation after a sudden increase of the glucose flux (Table 3):

1. The rate of oxygen consumption after a pulse is enhanced to approximately the same extent in Crabtree-positive and Crabtree-negative yeasts.

2. The rate of glucose consumption in a pyruvate decarboxylase-deficient mutant of $S$. cerevisiae is much lower than in cultures of wildtype strains, showing that pyruvate decarboxylase can exert a strong influence on the glycolytic flux.

3. During the first 30 minutes after the transition to glucose excess, the growth rate of $S$. cerevisiae, as calculated from cellular protein did not increase, indicating that an assimilatory bottleneck may also exist under these conditions. Dry weight measurements are misleading in 'transient state' experiments since various yeasts, notably Crab- tree-negative species, may accumulate large amounts of storage carbohydrates.

\section{Steady state kinetics of sugar metabolism}

In aerobic, glucose-limited chemostat cultures of the yeast Candida utilis, the residual substrate concentration can be described by Monod kinetics (Postma et al. 1988). Up to $\mu_{\text {max }}$, the metabolism of C. utilis is fully respiratory, and byproduct formation is negligible.

In (non-oscillating) aerobic, glucose-limited chemostat cultures of Saccharomyces cerevisiae, the residual glucose concentration was independent of the dilution rate up to a $\mu$ of $0.38 \mathbf{h}^{-1}$ (Postma et al. 1989b). Above this dilution rate, the glucose concentration increased. This change in the kinetics of glucose consumption is associated with a change in metabolism. Up to a dilution rate of $0.38 \mathrm{~h}^{-1}$, metabolism was oxidative, ethanol formation did not occur and the $\mathrm{qO}_{2}$ increased with increasing dilution rate (Fig. 4). Above this dilution rate, alcoholic fermentation occurred and the $\mathrm{qO}_{2}$ remained approximately constant (Postma et al. 1989b). The $\mathrm{qO}_{2}$ that is reached after careful adaptation of $S$. cerevisiae strains to high growth rates in sugar-limited chemostat cultures is frequently regarded as their maxi-



Fig. 5. Biomass yield (匹), specific oxygen consumption rate $(O)$ and glucose flux $(\nabla)$ as a function of benzoic acid concentration in aerobic, glucose-limited chemostat cultures of Saccharomyces cerevisiae, grown at a dilution rate of $0.10 \mathrm{~h}^{-1}$. 




Fig. 6. Effect of benzoic acid on specific oxygen consumption rates $\left(\mathrm{qO}_{2}\right)$ in glucose-limited, aerobic chemostat cultures of Saccharomyces cerevisiae, grown at various dilution rates. For each dilution rate the maximum $\mathrm{qO}_{2}$ was determined by growing cultures with a range of increasing benzoic acid concentrations in the reservoir vessel. The critical concentration of benzoic acid, at which $\mathrm{qO}_{2}$ was maximal, and above which alcoholic fermentation occurred, was dependent on the dilution rate. $(O), \mathrm{qO}_{2}$ in cultures grown in the absence of benzoic acid; ( $)$, maximum $\mathrm{qO}_{2}$ attained after addition of benzoic acid to the reservoir media of glucose-limited chemostat cultures.

mum respiratory capacity (Käppeli 1986). However, as is evident from the effect of weak acids on yeast cultures, the maximum respiratory capacity is very much dependent on cultivation conditions.

\section{Effect of weak organic acids on the glycolytic flux}

When weak organic acids such as acetic, propionic or benzoic acid are added to the reservoir medium of anaerobic, glucose-limited chemostat cultures of $S$. cerevisiae growing at a fixed dilution rate, the glycolytic flux increases: the rate of alcoholic fermentation is increased at the expense of biomass formation (Verduyn et al. 1990a, 1990b). This decrease of the growth efficiency is due to an increased energy requirement imposed by the net inward diffusion of the non-dissociated weak acid, caused by the transmembrane $\mathrm{pH}$ gradient. The protons that enter the cell must be expelled by the proton-pumping plas- ma membrane ATPase complex, to prevent intracellular acidification.

Also in aerobic chemostat cultures, the presence of non-metabolizable weak acids results in an enhancement of catabolism. As can be predicted from the model mentioned above, the uncoupling effect of benzoate on aerobic, glucose-limited chemostat cultures increased with decreasing culture $\mathrm{pH}$ (Verduyn 1991).

Under aerobic conditions, the increased glycolytic flux can be accomodated by respiration, which may attain very high rates (Fig. 5). However, above a 'critical' concentration of organic acid, a further sharp increase of the glycolytic flux was observed (Fig. 5), which was accompanied by the onset of alcoholic fermentation. The enhanced respiration provoked by benzoic acid was associated with an increase in mitochondrial volume to approximately one quarter of the cell volume (Verduyn et al. 1992a). Curiously, the degree to which benzoic acid could enhance respiration without triggering alcoholic fermentation was dependent on the specific growth rate: it decreased with increasing dilution rate (Fig. 6.). In cultures grown at dilution rates

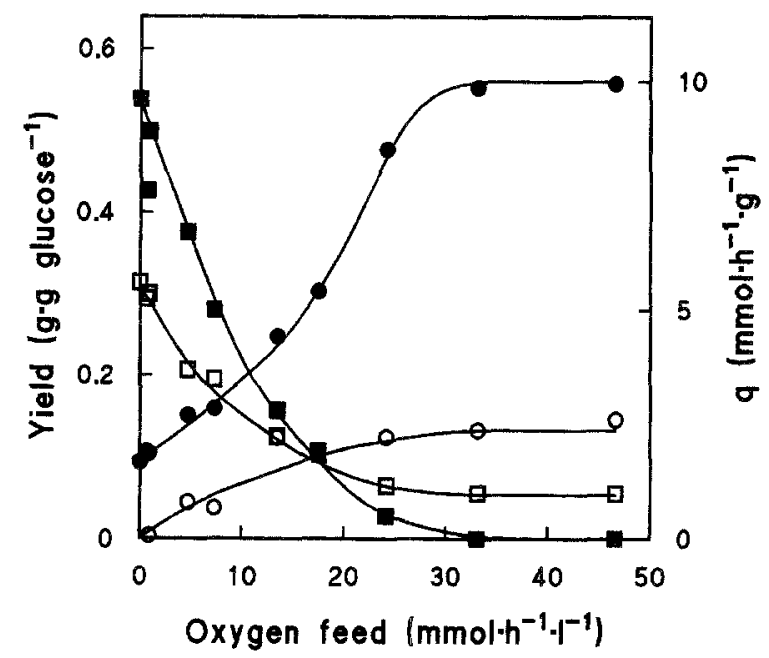

Fig. 7. Effect of oxygen feed on growth yield and glucose metabolism of Saccharomyces cerevisiae, grown at $\mathrm{D}=0.10 \mathrm{~h}^{-1}$ in glucose-limited chemostat cultures supplemented with Tween-80 and ergosterol. Cultures were grown in identical, 1 litre-working volume fermentors, at constant stirrer speed and gas flow through the cultures. Symbols: $\bullet$, biomass yield; $O, \mathrm{q} \mathrm{O}_{2} ; \square, \mathrm{q}$ glucose; $\boldsymbol{m}$, q ethanol. The zero value on the $\mathrm{x}$-axis points to the situation at which both the culture and the reservoir vessel were sparged with nitrogen gas. 
above $0.38 \mathrm{~h}^{-1}$, benzoate did not enhance $\mathrm{qO}_{2}$ but only decreased the cell yield with a concomitant rise in the specific rate of ethanol production (results not shown).

Also in aerobic cultures, the detrimental effect of organic acids on the growth efficiency is probably caused by an enhanced energy requirement, due to increased activity of the plasma membrane ATPase. Assuming that the presence of weak acids does not affect mitochondrial ATP formation (i.e. assuming that the $\mathrm{P} / \mathrm{O}$ ratio of mitochondrial respiration is not affected), it can be calculated that the additional ATP requirement for intracellular $\mathrm{pH}$ homeostasis is exactly the same under aerobic and anaerobic conditions (Verduyn 1992b).

\section{Effects of oxygen on growth kinetics}

In principle, all (facultatively) fermentative yeasts are able to generate ATP by substrate-level phosphorylation, and therefore do not depend on respiration to drive energy-requiring reactions. However, the physiological function of oxygen is not limited to its role as an electron acceptor for mitochondrial respiration. In fact, with the notable exception of Saccharomyces cerevisiae, none of the type species of the 75 yeast genera are able to grow under strictly anaerobic conditions with specific growth rates higher than $0.10 \mathrm{~h}^{-1}$ (Visser et al.1990). Even in the case of $S$. cerevisiae, anaerobic growth conditions impose additional growth factor requirements (in particular ergosterol, nicotinic acid and unsaturated fatty acids; Andreasen \& Stier 1953, 1954; Schatzmann 1975). The biosynthesis of these compounds involves oxygenation reactions and they therefore have to be included in the growth medium to allow anaerobic growth.

In Crabtree-negative yeasts, and in Crabtreepositive yeasts grown under sugar limitation, oxygen is a key parameter determining the rate of alcoholic fermentation. Oxygen feed can only be studied as a variable in chemostat cultures when the gas transfer properties of the cultures do not vary substantially among different steady states. In a series of recent studies, we have addressed this problem by using identical fermenters, and maintaining biomass concentrations, overall gas flow through the system and stirring speeds constant in the various steady states. An experiment of this type is given in Fig. 7, which shows the effect of varying oxygen feed on biomass yield and metabolic fluxes of glucose-limited chemostat cultures of $S$. cerevisiae (Weusthuis, unpubl.).

At high oxygen feeds, glucose metabolism by $S$. cerevisiae was fully respiratory and alcoholic fermentation was not observed. As the oxygen feed was decreased, alcoholic fermentation and respiration occurred simultaneously, and it was possible to grow S. cerevisiae under a double limitation of glucose and oxygen. In such glucose- and oxygen-limited cultures, an increase of the biomass concentration could be realized either by increasing the reservoir concentration of glucose (which led to increased alcoholic fermentation) or by increasing the oxygen feed (which allowed more respiration and, consequently, a higher growth yield on glucose). At all oxygen feeds investigated, the residual glucose concentration was below $0.5 \mathrm{mM}$. Since the experiments presented in Fig. 7 were carried out with a medium supplemented with Tween-80 and ergosterol, $S$. cerevisiae could also be grown strictly anaerobically (i.e. at zero oxygen feed).

When the type of experiment shown in Fig. 7 was carried out with Candida utilis, very similar results were obtained (data not shown). Alcoholic fermentation set in at approximately the same oxygen feed as with $S$. cerevisiae, and over a range of oxygen feeds, the cultures were both glucose- and oxygenlimited. Over this range, specific ethanol production rates were similar to those observed with $S$. cerevisiae. However, when the oxygen feeds were decreased to values below $3 \mathrm{mmol}^{-1} \mathrm{~h}^{-1}$, a threshold was reached and the residual concentration of glucose in the cultures increased. When the $C$. utilis cultures were sparged with nitrogen only, wash-out occurred. This confirmed earlier reports that $C$. utilis is unable to grow at growth rates above $0.05 \mathrm{~h}^{-1}$ under strictly anaerobic conditions (Visser et al. 1990). Apparently, alcoholic fermentation by nonSaccharomyces yeasts, like C. utilis, is confined to a narrow range of oxygen feeds. At very low oxygen feeds, growth (and, eventually, fermentation) becomes inhibited due to the intrinsic inability of 
these yeasts to grow anaerobically. At higher oxygen feeds, the glycolytic flux is preferentially directed towards respiration, thereby lowering the ethanol yield. Obviously, this will make it practically impossible to secure high ethanol yields in largescale industrial fermentations using non-Saccharomyces yeasts, e.g. for production of ethanol from sugar-containing waste streams such as whey and spent sulphite liquor or the fermentation of raw materials such as inulin and starch. Accurate dosing of oxygen, required to support fermentative growth of these yeasts, is not economically feasible in largescale industrial processes.

The inability of almost all facultatively fermentative yeasts to grow anaerobically in media that are adequate for $S$. cerevisiae is a hitherto unexplained phenomenon, which has very often been neglected in the literature dealing with alcoholic fermentation by non-Saccharomyces yeasts. For example, numerous papers have appeared on the fermentation of spent sulphite liquor (a waste stream from the paper industry, containing xylose and glucose) by these yeasts. In general, these publications focus on the oxygen requirement for the conversion of $x y-$ lose into ethanol, a phenomenon resulting from redox problems in the metabolism of xylose (Bruinenberg et al. 1984). However, oxygen is also required for the fermentation of glucose in such yeasts, since in the absence of oxygen they will not grow on glucose (although oxygen is not required for the conversion of glucose into ethanol).

In view of the potential advantages of non-Saccharomyces yeasts for the production of ethanol from sugar-containing raw materials and waste streams, the physiological background of their oxygen requirement for growth clearly merits research. Detailed knowledge in this field will make it possible to make a choice between two options:

1. transfer of genetic information needed for anaerobic growth from S. cerevisiae to non-Saccharomyces yeasts, or

2. the introduction of genes conferring non-Saccharomyces traits (e.g. broad substrate specificity) to $S$. cerevisiae.

Although oxygen limitation triggers alcoholic fermentation in facultatively fermentative yeasts, this is not a universal reaction. In some yeasts, certain sugars (often disaccharides) which support vigorous oxidative growth, cannot be fermented. In the literature, this effect has been termed the Kluyver effect (Sims \& Barnett 1978). We have studied this poorly understood phenomenon with respect to the utilization of maltose by the facultatively fermentative, Crabtree-negative yeast Candida utilis. Under aerobic or oxygen-limited conditions, this yeast is capable of respiration and growth on this disaccharide. However, even under oxygen-limited conditions as maintained in the experiments depicted in Fig. 7, production of ethanol from maltose by C. utilis could not be observed, although at identical oxygen feeds vigorous fermentation of glucose occurred (Weusthuis et al., unpubl.). In such cultures, the amount of maltose metabolized was strictly dependent on the oxygen feed. A satisfying explanation for the Kluyver effect is presently not available.

\section{Conclusions}

The kinetics of growth and sugar consumption by yeasts qualitatively and quantitatively depend on the yeast species, the nature and concentration of the sugar, the availability of oxygen and other environmental parameters such as the presence of weak acids. Knowledge about factors involved in the diversion of carbon fluxes into the formation of byproducts is still rather descriptive. Further research into the kinetics and regulation of key enzymes, especially those located at metabolic 'branchpoints', is required to identify targets and devise rational strategies for genetic redirection of metabolic fluxes (see the contribution of J.A. de Hollander). $\mathrm{Ob}$ viously, the relation between enzyme levels and metabolic fluxes should be studied under well-defined growth conditions, that allow the manipulation of metabolic fluxes. This may be accomplished, for example, by varying the oxygen feed or by inclusion of non-metabolizable weak acids in the reservoir medium of sugar-limited chemostat cultures growing at a fixed dilution rate. 


\section{References}

Andreasen AA \& Stier TJB (1953) Anaerobic nutrition of Saccharomyces cerevisiae. I. Ergosterol requirement for growth in a defined medium. J. Cell. Comp. Physiol. 41: 23-26.

Andreasen AA \& Stier TJB (1954) Anaerobic nutrition of Saccharomyces cerevisiae. II. Unsaturated fatty acid requirement for growth in a defined medium. J. Cell. Comp. Physiol. 43: 271-281

Bruinenberg PM, de Bot PHM, van Dijken JP \& Scheffers WA (1984) NADH-linked aldose reductase: the key to anaerobic alcoholic fermentation of xylose by yeasts. Appl. Microbiol. Biotechnol. 19: 256-260

D'Amore T \& Stewart GG (1987) Ethanol tolerance of yeast. Enzyme Microb. Technol. 9: 322-330.

Käppeli O (1986) Regulation of carbon metabolism in Saccharomyces cerevisiae and related yeasts. Adv. Microb. Physiol. 28: 181-209

Parulekar SJ, Semones GB, Rolf MJ, Lievense JC \& Lim HC (1986) Induction and elimination of oscillations in continuous cultures in Saccharomyces cerevisiae. Biotechnol. Bioeng. 28: 700-710

Petrik M, Käppeli O \& Fiechter A (1983) An expanded concept for the glucose effect in the yeast Saccharomyces uvarum: involvement of short- and long-term regulation. J. Gen. Microbiol. 129: 43-49

Postma E, Scheffers WA \& van Dijken JP (1988) Adaptation of the kinetics of glucose transport to environmental conditions in the yeast Candida utilis CBS 621: a continuous culture study. J. Gen. Microbiol. 134: 1109-1116

Postma E, Kuiper A, Tomasouw WF, Scheffers WA \& van Dijken JP (1989a) Competition for glucose between the yeasts Saccharomyces cerevisiae and Candida utilis. Appl. Env. Microbiol. 55: 3214-3220

Postma E, Verduyn C, Scheffers WA \& van Dijken JP (1989b) Enzymic analysis of the Crabtree effect in glucose-limited chemostat cultures of Saccharomyces cerevisiae. Appl. Environ. Microbiol. 55: 468-477

Rieger M, Käppeli O \& Fiechter A (1983) The role of a limited respiration in the incomplete oxidation of glucose by Saccharomyces cerevisiae. J. Gen. Microbiol. 129: 653-661

Romanos MA, Scorer CA \& Clare JJ (1992) Foreign gene expression in yeast: a review. Yeast 8: 423-488

Schatzmann H (1975) Anaerobes Wachstum von Saccharomyces cerevisiae. Thesis, Eidgenössische Hochschule Zürich, Switzerland

Sims AP \& Barnett JA (1978) The requirement of oxygen for the utilization of maltose, cellobiose and D-galactose by certain anaerobically fermenting yeasts (Kluyver effect). J. Gen. Microbiol. 106: 277-288

Van Dijken JP \& Scheffers WA (1986) Redox balances in the metabolism of sugars by yeasts. FEMS Microbiol. Rev. 32: 199-224

Van Dijken JP, van den Bosch JJ, Hermans L, de Miranda R \& Scheffers WA (1986) Alcoholic fermentation by 'non-fermentative' yeasts. Yeast 2:123-127

Van Urk H, Postma E, Scheffers WA \& van Dijken JP (1989a) Glucose transport in Crabtree-positive and Crabtree-negative yeasts. J. Gen. Microbiol. 135: 2399-2406

Van Urk H, Schipper D, Breedveld GJ, Mak PR, Scheffers WA \& van Dijken JP (1989b) Localization and kinetics of pyruvate-metabolizing enzymes in relation to aerobic alcoholic fermentation in Saccharomyces cerevisiae CBS 8066 and Candida utilis CBS 621. Biochim. Biophys. Acta 992: 78-86

Van Urk H, Voll WSL, Scheffers WA \& van Dijken JP (1990) Transient-state analysis of metabolic fluxes in Crabtree-positive and Crabtree-negative yeasts. Appl. Environ. Microbiol. 56: 281--287

Verduyn, C, Zomerdijk TPL, van Dijken JP \& Scheffers WA (1984) Continuous measurement of ethanol production by aerobic yeast suspensions with an enzyme electrode. Appl. Microbiol. Biotechnol. 19: 181-185

Verduyn C, Postma E, Scheffers WA \& van Dijken JP (1990a) Physiology of Saccharomyces cerevisiae in anaerobic glucoselimited chemostat cultures. J. Gen. Microbiol. 136: 395-403

Verduyn C, Postma E, Scheffers WA \& van Dijken JP (1990b) Energetics of Saccharomyces cerevisiae in anaerobic glucoselimited chemostat cultures. J. Gen. Microbiol. 136: 405-412

Verduyn C (1991) Physiology of yeasts in relation to growth yields. Antonie van Leeuwenhoek 60: 325-353

Verduyn C, Postma E, Scheffers WA \& van Dijken JP (1992a) Effect of benzoic acid on metabolic fluxes in yeasts: a continuous culture study on the regulation of respiration and alcoholic fermentation. Yeast 8: 501-517

Verduyn C (1992b) Energetic aspects of metabolic fluxes in yeasts. Thesis, Delft University of Technology, The Netherlands

Visser W, Scheffers WA, Batenburg-van der Vegte WH \& van Dijken JP (1990) Oxygen requirements of yeasts. Appl. Environ. Microbiol. 56, 3785-3792

Von Meyenburg HK (1969) Energetics of the budding cycle of Saccharomyces cerevisiae during glucose-limited aerobic growth. Arch. Microbiol. 66: 289-303 